\title{
BMJ Open Comparison of a preventive or curative strategy of fluid removal on the weaning of mechanical ventilation: a study protocol for a multicentre randomised open-label parallel-group trial
}

\author{
Martin Dres (D) , ${ }^{1}$ Candice Estellat, ${ }^{2}$ Jean-Luc Baudel, ${ }^{3}$ François Beloncle, ${ }^{4}$ \\ Julien Cousty, ${ }^{5}$ Arnaud Galbois, ${ }^{6}$ Laurent Guérin, ${ }^{7}$ Vincent Labbe, ${ }^{8}$ \\ Guylaine Labro (D) ${ }^{9}$ Jordane Lebut, ${ }^{10}$ Jean-Paul Mira, ${ }^{11}$ Gwenael Prat, ${ }^{12}$ \\ Jean-Pierre Quenot (D) , ${ }^{13}$ Armand Mekontso Dessap, ${ }^{14}$ The Reseau Européen de \\ Recherche en Ventilation Artificielle (REVA) research network
}

To cite: Dres M, Estellat C, Baudel J-L, et al. Comparison of a preventive or curative strategy of fluid removal on the weaning of mechanical ventilation: a study protocol for a multicentre randomised open-label parallel-group trial. BMJ Open 2021;11:e048286. doi:10.1136/ bmjopen-2020-048286

- Prepublication history for this paper is available online. To view these files, please visit the journal online (http://dx.doi. org/10.1136/bmjopen-2020048286).

Received 22 December 2020 Accepted 29 July 2021

Check for updates

(C) Author(s) (or their employer(s)) 2021. Re-use permitted under CC BY-NC. No commercial re-use. See rights and permissions. Published by BMJ.

For numbered affiliations see end of article.

Correspondence to

Dr Martin Dres;

martin.dres@aphp.fr

\section{ABSTRACT}

Introduction Fluid overload is associated with a poor prognosis in the critically ill patients, especially at the time of weaning from mechanical ventilation as it may promote weaning failure from cardiac origin. Some data suggest that early administration of diuretics would shorten the duration of mechanical ventilation. However, this strategy may expose patients to a higher risk of haemodynamic and metabolic complications. Currently, there is no recommendation for the use of diuretics during weaning and there is an equipoise on the timing of their initiation in this context.

Methods and analysis This study is a multicentre randomised controlled trial comparing two strategies of fluid removal during weaning in 13 French intensive care units (ICU). The preventive strategy is initiated systematically when the fluid balance or weight change is positive and the patients have criteria for clinical stability; the curative strategy is initiated only in case of weaning failure documented as of cardiac origin. Four hundred and ten patients will be randomised with a 1:1 ratio. The primary outcome is the duration of weaning from mechanical ventilation, defined as the number of days between randomisation and successful extubation (alive without reintubation nor tracheostomy within the 7 days after extubation) at day 28 . Secondary outcomes include daily and cumulated fluid balance, metabolic and haemodynamic complications, ventilator-associated pneumonia, weaning complications, number of ventilatorfree days, total duration of mechanical ventilation, the length of stay in ICU and mortality in ICU, in hospital and, at day 28. A subgroup analysis for the primary outcome is planned in patients with kidney injury (Kidney Disease: Improving Global Outcomes class 2 or more) at the time of randomisation.

Ethics and dissemination The study has been approved by the ethics committee (Comité de Protection des Personnes Paris 1) and patients will be included after informed consent. The results will be submitted for publication in peer-reviewed journals.
Strengths and limitations of this study

This large randomised controlled trial may help establish strong recommendations with a high level of evidence on routine clinical practice during weaning from mechanical ventilation.

- The sample size of this trial has been designed to have the power to show an absolute reduction of the weaning duration.

- The individual study assignments of the patients will not be masked (given the characteristics of the two strategies under evaluation, a double-blind trial, was deemed not possible).

Trial registration number NCT04050007. Protocol version V.1; 12 March 2019.

\section{INTRODUCTION}

Background and rationale

Mechanical ventilation is a cornerstone treatment for the critically ill, which is however associated with complications. A major objective is, therefore, to separate patients from the ventilator as quickly as possible, but without exposing them to the risk of extubation failure. ${ }^{1}$ Pulmonary oedema is a frequent cause of difficult weaning and extubation failure, up to $60 \%$ in recent series. ${ }^{23} \mathrm{~A}$ positive fluid balance has also been identified as an important risk factor for difficult weaning and extubation failure. ${ }^{4-6}$ More generally, a positive fluid balance is associated with a poor prognosis in different populations of critically ill patients such as those with acute respiratory distress syndrome, ${ }^{7}$ acute kidney injury $^{8}$ or septic shock. ${ }^{9} \mathrm{~A}$ 'de-escalation' or 
'deresuscitation' strategy consisting of early fluid removal to obtain a negative fluid balance as soon as the haemodynamic state is stabilised has been suggested. ${ }^{6}$ Nevertheless, the implementation of such strategy faces many difficulties in terms of monitoring methods and therapeutic targets. In patients with acute respiratory distress syndrome ${ }^{7}$ or sepsis, ${ }^{10}$ a conservative fluid management strategy is associated with an improvement in haemodynamic parameters despite an increase in urine output. The conservative approach also results in a significant improvement in oxygenation and a trend towards a shorter duration of artificial ventilation and ICU stay. ${ }^{7}$ During the specific phase of weaning from mechanical ventilation, a randomised trial (BMW trial) demonstrated that a strategy of fluid removal guided by measurement of the plasmatic B-type natriuretic peptide significantly reduced the duration of weaning. ${ }^{11}$ Fluid overload is clearly associated with an increased risk of extubation failure and the increase in cardiac preload is one of the main mechanisms leading to weaning-induced cardiac failure, especially in patients with chronic cardiac disease. Thus, in case of obvious fluid overload, obtaining a negative fluid balance before the next spontaenous breathing trial (SBT) is a reasonable option that has not, however, been yet evidenced. ${ }^{12}$

\section{Hypothesis}

The hypothesis of the present study is that a preventive and systematic strategy of fluid removal (initiated before the weaning phase, as soon as the patients are stabilised) would shorten the duration of weaning from mechanical ventilation as compared with a strategy of curative fluid removal (initiated only in case of weaning failure from cardiovascular origin).

\section{Objectives}

\section{Primary objective}

The main objective is to evaluate the impact of a strategy of preventive fluid removal (as compared with a curative fluid removal) on the duration of weaning from mechanical ventilation.

\section{Secondary objectives}

To compare the following endpoints between the two groups:

1. metabolic complications (hypernatremia, hypokaliaemia, acute kidney injury) at day 28.

2. Haemodynamic complications at day 28.

3. Daily and cumulated fluid balance at day 28 .

4. Weaning complications at day 28 , including failure of the first spontaneous breathing trial, reintubation within the 7 days after extubation, ${ }^{13}$ use of unplanned non-invasive ventilation or high flow oxygen therapy within the 7 days after extubation, tracheostomy.

5. Number of ventilator-free days within the 14 and 28 days following randomisation.
6. Total duration of mechanical ventilation from intubation to successful extubation (patient alive without invasive ventilation within 7 days after extubation).

7. Ventilator-associated pneumonia.

8. Length of stay in ICU.

9. Mortality in ICU, in hospital and at day 28 .

10. Duration of weaning according to acute kidney injury at the time of randomisation.

\section{METHODS AND ANALYSIS \\ Trial design}

This study is a multicentre, randomised (1:1) open-label trial with two arms, to test the superiority of a preventive fluid removal strategy, as compared with a curative fluid removal strategy.

The trial accords with the Standard Protocol Items: Recommendations for Interventional Trial guidelines.

\section{Study setting}

This study will take place in at least 13 ICUs in France. Patients flowchart is detailed in figure 1.

\section{Eligibility criteria}

Inclusion criteria

Adult patients admitted in ICU will be eligible as soon as they meet all of the following criteria:

1. Age $>18$ years.

2. Intubation and mechanical ventilation $\geq 24$ hour.

3. Positive cumulative fluid balance or increase in body weight since admission.

4. Clinical stability as defined by: (1) stable oxygenation $\left(\mathrm{SpO}_{2} \geq 90 \%\right.$ with $\mathrm{FiO}_{2} \leq 50 \%$ and Positive end expiratory pressure (PEEP) $\leq 8 \mathrm{~cm} \mathrm{H_{2 }} \mathrm{O}$ ), (2) stable haemodynamics (no vasopressors and no fluid expansion within the last 12 hours), (3) sedation was stopped or decreased within the last 48 hours with stable neurologic state (with Richmond Agitation Sedation Scale (RASS) $\geq-4$ or Ramsay $\leq 5$ ), (4) temperature is $>36^{\circ} \mathrm{C}$ and $<39^{\circ} \mathrm{C}$.

5. Informed consent is signed by the patient or next of kin or emergency procedure.

\section{Non-inclusion criteria}

Patients fulfilling one of the following criteria will not be included:

1. Extracorporeal membrane oxygenation.

2. Pregnancy or breast feeding.

3. Allergy to furosemide, sulfamides or spironolactone.

4. Tracheotomy.

5. Hydrocephaly.

6. Acute right ventricle failure.

7. Cardiac arrest with estimated poor prognosis.

8. Already enrolled in an interventional study on weaning from mechanical ventilation.

9. Guillain-Barre syndrome, myasthenia gravis.

10. Planned extubation on the day of inclusion.

11. Criteria of clinical stability (as described above in inclusion criteria) present since more than 24 hours. 


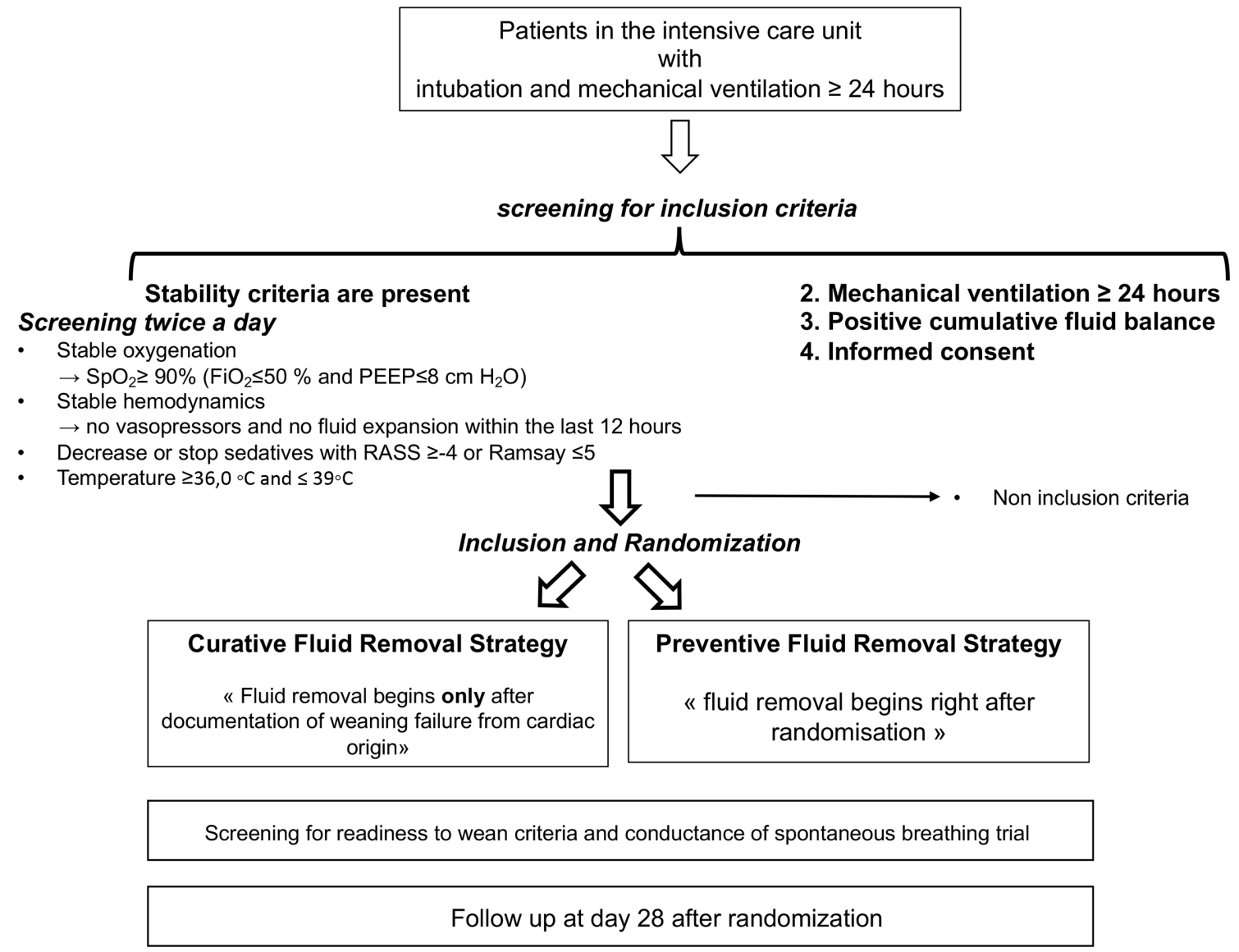

Figure 1 Flowchart of the patient and study design.

12. Natremia $>150 \mathrm{mEq} / \mathrm{L}$, kaliemia $<3.5 \mathrm{mEq} / \mathrm{L}$, metabolic alkalosis with $\mathrm{pH}>7.5$.

13. Administration of iodinated contrast within the last 6 hours.

14. Ongoing or planned use of artificial kidney within the next 48 hours.

15. No affiliation to the health insurance system.

16. Patient under curatorship.

17. Imprisoned patient.

\section{Intervention}

Preventive fluid removal strategy

Preventive fluid removal will be initiated right after the randomisation in all patients with a positive fluid balance or with an increase in weight since the ICU admission.

\section{Curative fluid removal strategy}

The initiation of fluid removal will be considered by the attending physician only in case of difficult weaning deemed of cardiac origin (failure of the spontaneous breathing trial or failure of the decrease in the ventilator support, if this failure is associated with one of the following three categories of signs of weaninginduced pulmonary oedema). First, clinically, weaninginduced pulmonary oedema will be suspected in patients presenting three among five of the following conditions: (1) previous cardiovascular disease, (2) previous respiratory disease, (3) sudden onset of severe hypertension during the spontaneous breathing trial, (4) increase in heart rate and (5) bilateral crackling auscultation. Second, by using echocardiography (performed by the attending physician), weaning-induced pulmonary oedema will be suspected in case of increase in left ventricle filling pressure at the end of the spontaneous breathing trial. Third, biologically, weaning-induced pulmonary oedema will be suspected by using biomarkers (B-type natriuretic peptide or plasma protein concentration) (figure 2). ${ }^{12}$ 14-18

\section{Fluid removal protocol}

Both groups will follow the same protocol of fluid removal, involving fluid intake restriction, diuretic administration and electrolyte compensation (only the timing will be different). The daily total volume of fluids will be limited $(<1500 \mathrm{~mL} / 24$ hours $)$ and sodium intakes will be restricted to the minimum (nutrition and drugs). Furosemide will be administered (as intravenous bolus doses of 0-30 mg every 3 hours, to achieve a target urine output of $4.5-9 \mathrm{~mL} / \mathrm{Kg} / 3$ hours) (see table 1 ). Fluid intake restriction and diuretic administration will be continued for at least 48 hours after extubation. Sodium, potassium, urea, creatinine and arterial blood gases will be monitored daily.

Recommendations will be given to prevent and/or treat possible adverse events related to diuretic treatment, as detailed hereafter 


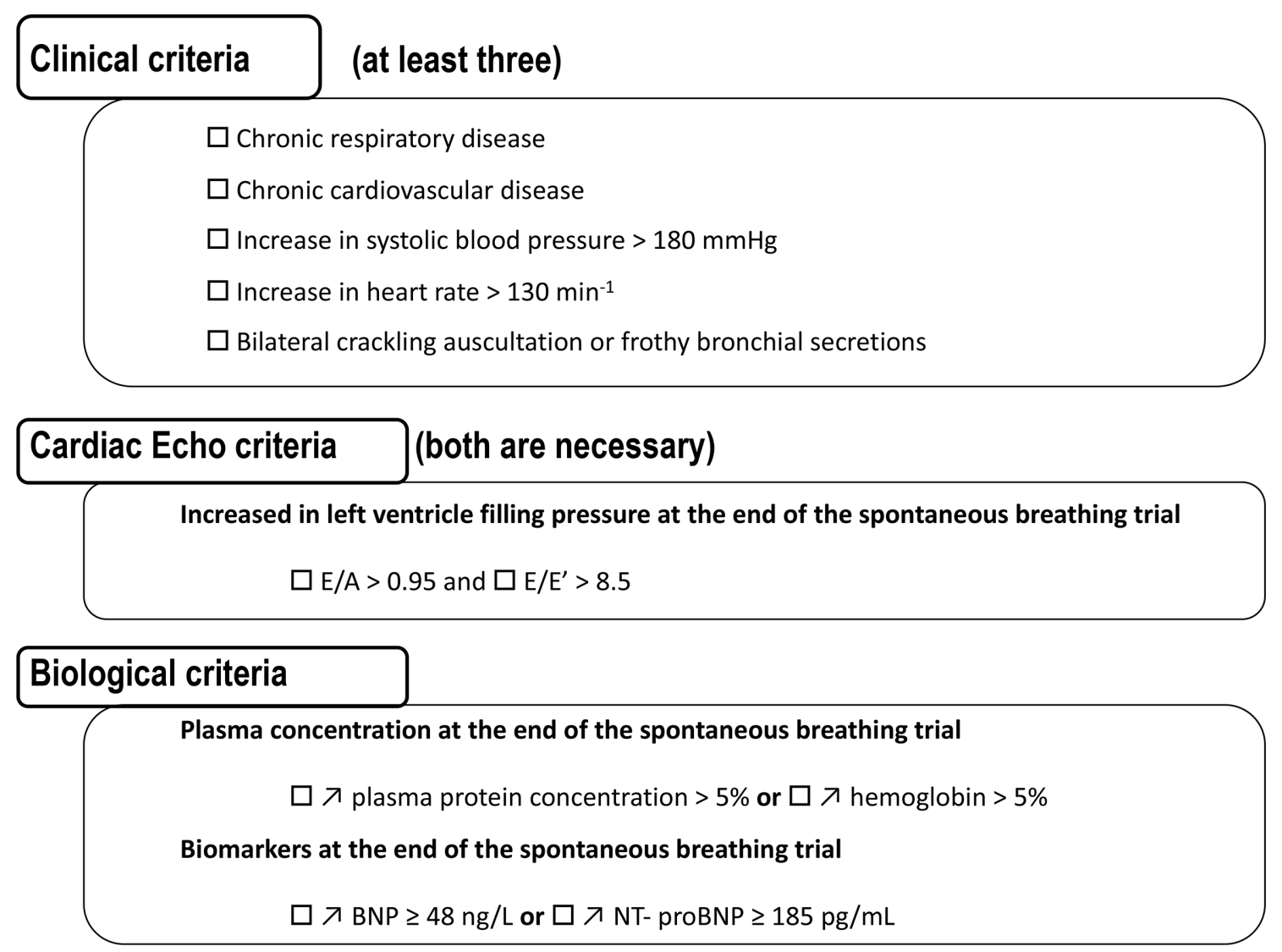

Figure 2 Criteria suggesting weaning failure from cardiac origin. BNP, brain natriuretic peptide; E/A, early (E) over late (A) diastolic wave velocities at the mitral valve; E/e', E wave over tissue doppler early (e') wave velocity at the lateral mitral valve annulus.

- If urine output exceeds $36 \mathrm{~mL} / \mathrm{kg} / 12$ hours or blood potassium is $<4.0 \mathrm{mEq} / \mathrm{L}$ while receiving diuretics, blood electrolytes will be checked within the next 12 hours.

- In the event of metabolic alkalosis with furosemide, acetazolamide will be added $(250 \mathrm{mg}$ every 8 hours if $\mathrm{pH}>7.45$ or $500 \mathrm{mg}$ every 8 hours if $\mathrm{pH}>7.50$ ) in the absence of contraindications (history of hypersensitivity to acetazolamide or sulphonamides; severe hepatic, renal or adrenal insufficiency or history of renal lithiasis).

- If blood potassium is $<4.5 \mathrm{mEq} / \mathrm{L}$ during diuretic therapy, supplemental potassium will be given $(\geq 4 \mathrm{~g} /$ day if blood potassium is $<4.0 \mathrm{mEq} / \mathrm{L}$ or $\geq 3 \mathrm{~g} /$ day if blood potassium is between $4.0 \mathrm{mEq} / \mathrm{L}$ and 4.4

\begin{tabular}{lll|}
\hline Table 1 & Algorithm of diuretics administration \\
\hline $\begin{array}{l}\text { Initial dose of } \\
\text { furosemide }(\mathbf{m g})\end{array}$ & $\begin{array}{l}\text { Urine output } \\
(\mathbf{m L} / \mathbf{k g} / 3 \text { hours) }\end{array}$ & $\begin{array}{l}\text { Subsequent doses } \\
\text { of furosemide }(\mathbf{m g})\end{array}$ \\
\hline 20 & $<4.5$ & 30 \\
$4.5-6$ & 20 \\
$6-7.5$ & 15 \\
$7.5-9$ & 10 \\
& $>9$ & 0 \\
\hline
\end{tabular}

$\mathrm{mEq} / \mathrm{L})$. Magnesium supplements $(\geq 1.5 \mathrm{~g} /$ day $)$ will be given routinely during diuretic treatment.

- In case of acute kidney injury under diuretic treatment, the management will depend on the KDIGO stage:

Stage 1: echocardiography will be considered to search for left ventricle dysfunction (enabling dobutamine therapy), hypovolaemia or congestion.

Stage 2: echocardiography will be considered (see above) and the fluid removal protocol will be temporarily suspended.

Stage 3: the fluid removal protocol will be stopped.

- If blood sodium exceeds $150 \mathrm{mEq} / \mathrm{L}$, hypotonic solutions may be given to increase the daily fluid intake above $500 \mathrm{~mL}$ (no salt intake).

- If iodinated contrast agent injection is expected to be needed, diuretic administration will be suspended 6 hours before and 6 hours after the infusion.

The other conditions requiring furosemide discontinuation will be as follows: metabolic alkalosis with arterial $\mathrm{pH}>7.55$, blood potassium $<3.0 \mathrm{mEq} / \mathrm{L}$, blood sodium $>155 \mathrm{mEq} / \mathrm{L}$, acute kidney injury (stage 2 KDIGO), urine output $>9 \mathrm{~mL} / \mathrm{kg} / 3$ hours and hypotension requiring fluid bolus or vasopressor therapy. When diuretic treatment will be stopped because of one of these abnormal findings, it can be reinstituted after correction 
of the abnormal value, in accordance with the inclusion and non-inclusion criteria. The first furosemide dose after reinstitution will be half the last dose administered.

\section{Strategy of weaning, extubation and prevention of extubation failure}

In both groups, the weaning process and postextubation management will be protocolised based on national guidelines. ${ }^{19}$ Use of sedatives and analgesia will be left at the discretion of the physician as per the local protocol, but daily cumulative doses will be collected. The presence of the following readiness to wean criteria will be screened every morning and will trigger a weaning trial (either T-tube or pressure support trial with pressure support set at $7 \mathrm{cmH}_{2} \mathrm{O}$ and zero-end expiratory pressure for a duration between $30 \mathrm{~min}$ and $60 \mathrm{~min}$ ):

1. Patients under pressure support with positive-end expiratory pressure $\leq 5 \mathrm{cmH}_{2} \mathrm{O}, \mathrm{FiO}_{2} \leq 40 \%, \mathrm{SpO}_{2} \geq 90 \%$ and respiratory rate $<30 / \mathrm{min}$ without signs of respiratory muscles labouring.

2. No vasopressors, heart rate $<130 / \mathrm{min}$ and systolic blood pressure between $95 \mathrm{~mm} \mathrm{Hg}$ and $160 \mathrm{~mm} \mathrm{Hg}$.

3. Temperature between $36.5^{\circ} \mathrm{C}$ and $38.5^{\circ} \mathrm{C}$.

4. Sedatives stopped with RASS score between -1 and +1 .

5. Audible cough during tracheal suctioning or spontaneously.

6. Need for less than three suctioning during the last 4 hours.

In case of success of the weaning trial, investigators will be encouraged to proceed with extubation on the day of the trial. In case of failure of the weaning trial, a new trial will be performed daily as long as weaning criteria are met, until success and extubation. Failure of the weaning trial will be defined according to the usual criteria from the International Conference Consensus on Weaning, ${ }^{20}$ as development during the trial of any of the following events: (1) respiratory rate $>35$ breaths/ min, (2) increased accessory muscle activity, (3) $\mathrm{SpO}_{2}$ persistently below $90 \%$ (or below $88 \%$ in case of underlying chronic lung disease) on $\mathrm{FiO}_{2} \geq 0.4$ or at least $6 \mathrm{~L} /$ min of oxygen, (4) haemodynamic instability defined as heart rate persistently above 140 beats/min or systolic blood pressure $<90 \mathrm{~mm} \mathrm{Hg}$ or $>180 \mathrm{~mm} \mathrm{Hg}$, with signs of hypoperfusion (appearance of cyanosis or mottling), (5) depressed mental status or agitation.

After extubation, prophylactic use of non-invasive ventilation alternating with high-flow nasal oxygen between non-invasive ventilation sessions will be recommended in patients with risk factors for extubation failure. ${ }^{13} 1921$

\section{Criteria for reintubation}

To ensure the consistency of indications across sites and reduce the risk of delayed intubation, investigators will be suggested to reintubate patients if one of the following criteria is fulfilled: (1) cardiac arrest, (2) decreased level of consciousness associated with respiratory pauses, (3) inability to cope with abundant bronchial secretions, (4) aspiration, (5) shock state with the need for vasopressor, (6) respiratory failure, (7) unplanned surgery.

\section{Outcomes}

\section{Primary outcome}

The primary outcome is the duration of weaning as defined by the time elapsed between the day of randomisation and the day of successful extubation (patient alive, with no tracheostomy and no reintubation within the 7 days following extubation) until day 28. If the patient dies without being extubated within 28 days after randomisation, the patient will count as weaning failure, and the duration of weaning will be of 28 days.

\section{Secondary outcomes}

Secondary outcome will include the following:

1. Percentage of patients with metabolic complications among: hypernatremia $>150 \mathrm{mEq} / \mathrm{L}$, hypokaliaemia $<2.5 \mathrm{mEq} / \mathrm{L}$, acute renal failure with KDIGO stages 2 and 3 , before day 28 .

2. The occurrence before day 28 of any haemodynamic complications among hypotension with systolic blood pressure $<90 \mathrm{~mm} \mathrm{Hg}$, introduction or increase in vasopressors dose, use of fluid expansion, atrial fibrillation, ventricular fibrillation.

3. Daily and cumulated fluid balance as assessed by the difference between all fluid intakes (nutrition, hydration, drugs, perfusions) and fluid outputs (urine output, digestive suctioning, pleural drainage).

4. The rate of patients who failed the first spontaneous breathing trial, as defined by the International Conference Consensus on Weaning ${ }^{20}$ (see above).

5 . The rate of reintubation within the 7 days after extubation.

6. The rate of use of unplanned non-invasive ventilation and high flow oxygen.

7. The rate of tracheostomy.

8. The number of days alive and free from mechanical ventilation (including intubation and non-invasive ventilation) between randomisation and day 14 and day 28.

9. The total number of days of mechanical ventilation (from intubation to successful extubation), until day 28.

10. The percentage of patients with ventilator-associated pneumonia. ${ }^{22}$

11. The duration of ICU and hospital stays, until day 28.

12. Percentage of deaths in the ICU, in the hospital and at day 28 among patients.

\section{Sample size and its statistical justification}

A statistician calculated the sample size by estimating by simulation (2000 data sets) under a Weibull distribution using the following hypotheses derived from the BMW trial $^{11}$ : a cumulative incidence of successful extubation of $60 \%$ by day 7 and $80 \%$ by day 28 taking into account a competitive risk of death of $13 \%$ by day 7 and $16 \%$ by day 28. We determined that the enrolment of 410 patients 
(205 patients per group) would provide a power of $90 \%$ to show an absolute reduction of weaning duration of 1.6 days ( 4.9 days for the curative strategy versus 3.3 days for the preventive strategy) with a two-sided alpha level of 0.05 using a likelihood ratio test in a Fine \& Gray model with a time interaction term.

\section{Recruitment}

The expected initial duration of patient enrolment is 2 years, starting in February 2020. The chronogram of the study is as follows:

1. End of 2018: national grant award.

2. 2019: approval by an independent ethics committee.

3. 2020-2021: inclusion of patients.

4. 2021-2022: end of inclusions, monitoring of participating centres and queries to investigators; cleaning and closure of the database; blind review to determine protocol violation, to define intention-to-treat and perprotocol analysis populations.

5. 2022-2023: data analysis, writing of the manuscript and submission for publication.

As of 21 December 2020, 84 patients have been randomised in the study.

\section{Assignment of intervention and data collection}

After obtaining consent from the patient or her/his relative, all inclusion/exclusion criteria will be checked by the investigator before randomisation. Randomisation will be stratified on centre and left ventricle ejection fraction (>or $\leq 45 \%$ ) at the time of randomisation and carried out by connecting to the centralised electronic-case report form (e-CRF website 'Cleanweb' provided by Telemedicine technologies. Data will be collected on the e-CRF by a trained investigator or research assistant at each centre.
Patient follow-up and data collected are detailed in the study flowchart (table 2).

\section{Statistical methods}

All the analyses will be performed by the study statistician according to a predefined statistical analysis plan, using $\mathrm{R}$ software ( $\mathrm{R}$ Foundation for Statistical Computing, Vienna, Austria) or Statistical Analysis System (SAS) latest versions. A two-tailed $\mathrm{p}$ value of less than 0.05 will be considered as indicating statistical significance.

In accordance to the CONsolidated Standards Of Reporting Trials (CONSORT) statement, a flow diagram will describe the progress of patients of the two groups through the phases of the trial (enrolment, intervention allocation, intervention received, follow-up and data analysis). The analysis will be performed on an intention-totreat basis. In case of premature stop or withdrawal from the study, patients would not be substituted. Missing value will be described and, according to nature and frequency, multiple imputation methods will be used. A per-protocol analysis will be held as sensitivity analysis, excluding patients wrongly randomised or who did not receive allocated intervention.

Comparative analysis will systematically be done with (main analysis) and without adjustment on randomisation stratification factors. No interim analysis is planned.

\section{Descriptive analysis}

The continuous variables will be summarised with the classic parameters of descriptive analysis (median, IQRs and extreme values or mean and SD), while indicating the number of missing data. Categorical variables will be presented in the form of absolute frequency and percentage in each modality. Censored variables will

Table 2 Flowchart of timing in collection of different variables

\begin{tabular}{|c|c|c|c|c|}
\hline Procedures and assessments & Screening & $\begin{array}{l}\text { Inclusion visit and } \\
\text { randomisation (day 0) }\end{array}$ & Daily visits & Study end (day 28) \\
\hline Inclusion and non-inclusion criteria & $x$ & & & \\
\hline \multicolumn{5}{|l|}{ Enrolment } \\
\hline Information & & $x$ & & \\
\hline Consent & & $x$ & & \\
\hline \multicolumn{5}{|l|}{ Intervention } \\
\hline Curative strategy & & & & o \\
\hline \multicolumn{5}{|l|}{ Assessments } \\
\hline Characteristics of the patient ${ }^{\star}$ & & $\mathrm{x}$ & $x$ & $x$ \\
\hline $\begin{array}{l}\text { Characteristics of ventilation and } \\
\text { fluid management } †\end{array}$ & & $x$ & $x$ & $x$ \\
\hline
\end{tabular}

${ }^{*}$ Characteristics of the patient include age, gender, height, weight, severity score indicated by the Simplified Acute Physiological Score II and the Sepsis-related Organ Failure Assessment score, underlying chronic cardiac or respiratory disease, date and reason for admission/intubation, duration of intubation prior randomisation, weaning characteristics.

†Characteristics of ventilation and fluid management include ventilator settings, blood samplings, urine output, fluid intakes, cardiac echo, chest X-ray. ICU, intensive care unit. 
be described by Kaplan-Meier plots. Variables will be described for the whole group and separately for each arm.

\section{Analysis of the primary outcome}

The duration of weaning from mechanical ventilation, as defined by the number of days between randomisation and successful extubation at day 7 (patients alive, no reintubation, no tracheostomy), will be compared between the two groups using a Fine \& Gray model, with a likelihood ratio test, in order to take into account the competitive risk of death. A time-interaction term will be used to take into account the proportional hazard assumption violation. Subdistribution HR associated with the preventive strategy will be estimated with its $95 \%$ CI.

\section{Analysis of secondary outcomes}

Rate of metabolic and haemodynamic complications, rate of ventilator-associated pneumonia, extubation success, reintubation, tracheostomy and mortality at the various predefined times will be compared between the two groups using logistic models. Mean daily and cumulative fluid balance calculated until the ICU discharge and ventilatory free days at day 14 and day 28 will be compared between groups using a linear regression. Weaning duration and lengths of stay will be compared between the two treatment groups using similar models as for primary outcome. The effect of the preventive strategy on mortality (in ICU, at day 28 and at in hospital) will be evaluated using Cox models.

\section{Predetermined subgroup analysis}

The effect of acute kidney injury prior to randomisation on the efficacy of the preventive strategy of fluid removal will be investigated by introducing an interaction term in the Fine \& Gray model. If significant, a subdistribution HR associated with the preventive strategy will be estimated.

\section{Data monitoring}

The trial will be overseen by a steering committee (principal investigator, senior investigator and methodologist) regarding the progression and monitoring of the study. Research assistants will regularly monitor all the centres on site to check adherence to the protocol and the accuracy of the data recorded. An investigator at each centre will be responsible for daily patient screening, enrolling patients in the study, ensuring adherence to the protocol and completing the electronic case report form. Since both strategies are currently used in routine practice, no data safety monitoring board was required by the ethical committee.

\section{ETHICS AND DISSEMINATION}

\section{Consent to participate}

The patient will be included after having provided a written informed consent to the investigator. If the patient is not able to understand the information given, he/ she can be included if the same procedure is completed with a next of kin. Eligible patients who will be unable to receive information and for whom a substitute decisionmaker would not be present may be included through a process of deferred consent. After the patient's recovery, she/he will be asked if she/he agrees to continue the trial. The protocol has been approved by an independent Ethical Committee (Comité de Protection des Personnes Paris 1).

\section{Confidentiality}

Data will be handled according to French law on data protection and European General Data Protection Regulation. All original records will be archived at trial sites for 15 years.

\section{Declaration of interest}

This study was funded by a grant from the French Ministry of Health obtained in 2018 (Programme Hospitalier de Recherche Clinique). The sponsor is Assistance Publique-Hôpitaux de Paris, AP-HP (Délégation à la Recherche Clinique et à l'Innovation, DRCI).

\section{Access to data}

Investigators will make available the documents and individual data strictly required for monitoring, quality control and audit of the study to dedicated persons, in accordance with law.

\section{Dissemination policy}

Findings will be published in peer-reviewed journals and presented at national and international meetings. Communications, reports and publication of the results of the study will be placed under the responsibility of the principal investigator-coordinator of the study and the steering committee. Reporting will follow CONSORT statement and rules of publication will follow the international recommendations according to The Uniform Requirements for Manuscripts (ICMJE, April 2010).

\section{Individual participant data sharing statement}

Data are available on reasonable request. The procedures carried out with the French data privacy authority (Commission Nationale de l'Informatique et des Libertés) do not provide for the transmission of the database, nor do the information and consent documents signed by the patients. Consultation by the editorial board or interested researchers of individual participant data that underlie the results reported in the article after deidentification may nevertheless be considered, subject to prior determination of the terms and conditions of such consultation and in respect for compliance with the applicable regulations.

\section{Patient and public involvement}

Patients and public were not involved in the study design. Participants will have access to the findings of the study on request. 


\section{DISCUSSION}

Up to now, there are no randomised controlled trials available on the timing of initiation of fluid removal during weaning. Liberation from mechanical ventilation is a crucial process that can be associated with complications that can delay extubation. ${ }^{1}$ Increasing the duration of mechanical ventilation at the time of weaning is associated with an increased risk of mortality. ${ }^{23}$ The objective of the present study is to reduce the duration of weaning, a major outcome for the critically ill patients highlighting the relevant dividends of this trial for the future. Among the numerous factors associated with weaning failure, the role of fluid overload has been highlighted in several reports. ${ }^{4} 57624$ In several studies, successful weaning was achieved after diuretic treatment in patients who had evidence of weaning-induced pulmonary oedema. ${ }^{21624}$ However, the timing of fluid removal is still a matter of discussion and there are currently no guidelines that recommend the use of diuretics at the initiation of weaning. Of note, a recent retrospective study found that a negative fluid balance on day 3 of ICU stay was associated with lower 30-day mortality whether occurring spontaneously or achieved with deresuscitative measures. ${ }^{6}$ A study examining the relationship between loop diuretic use and hospital mortality in critically ill patients with vasopressor support found a reduced mortality in patients who received diuretics. ${ }^{25}$ Nevertheless, another study did not confirm this association on 13000 patients from a national database. ${ }^{26}$

There are several advantages for using a preventive fluid removal strategy as tested in the current trial. First, it may allow to achieve a negative fluid balance before starting the spontaneous breathing trial, a condition that may reduce the risk of weaning failure from cardiac origin. ${ }^{21124}$ Second, as a positive fluid balance has been associated with worse outcomes, ${ }^{72728}$ it is tempting to limit it early in the ICU course and if possible to achieve a negative fluid balance as soon as the patient is stabilised. However, such a preventive strategy may be associated with some complications, like dehydratation with renal failure, ${ }^{8}$ electrolyte imbalances, ${ }^{726}$ hypotension and atrial fibrillation. ${ }^{29}$ Given this equipoise, the current trial aims at comparing the preventive fluid removal strategy with a more conservative strategy initiated only in case of weaning failure from cardiac origin. ${ }^{12}$ Before starting diuretics in patients of the curative group, investigators of the present trial will have to document the presence of weaning-induced pulmonary oedema via clinical signs, echocardiography markers ${ }^{18}$ or biomarkers ${ }^{15} 16$ that have been proposed in the literature. ${ }^{12}$

The use of diuretics will be standardised with a robust and validated algorithm. ${ }^{11}$ In addition, preventive measures will be associated for a careful management of fluid removal and electrolytes. To reduce biases and ensure a satisfactory comparison between preventive and curative groups, the weaning process will be protocolised based on the current national guidelines. ${ }^{19}$ Of note, spontaneous breathing trial will be performed based on a daily screening for readiness to wean criteria and preventive strategies for extubation failure will be applied based on the most recent evidences. ${ }^{132130}$

In summary, this trial is an open-label randomised controlled trial testing two strategies for fluid removal (preventive vs curative) to reduce the duration of weaning from mechanical ventilation. This kind of comparison has never been performed before. Therefore, this trial may help establish international recommendations with a high level of evidence for weaning from mechanical ventilation to eventually improve the outcomes of patients exposed to mechanical ventilation.

\section{Author affiliations}

${ }^{1}$ Service de Médecine intensive Réanimation, APHP.Sorbonne Université, Hôpital Pitie Salpêtriere, Paris, France

²Département de Santé Publique, APHP.Sorbonne Université, Hôpital Pitie Salpetriere, Paris, Île-de-France, France

${ }^{3}$ Service de Médecine intensive Réanimation, APHP.Sorbonne Université, Hôpital Saint Antoine, Paris, Île-de-France, France

${ }^{4}$ Service de Médecine intensive Réanimation et médedine hyperbare, CHU Angers, Angers, Pays de la Loire, France

${ }^{5}$ Service de Réanimation Polyvalente, CHU de La Réunion Sites Sud Saint-Pierre, Saint-Pierre, Réunion

${ }^{6}$ Service de Réanimation polyvalente, Hôpital Claude Galien, Quincy-sous-Senart, France

${ }^{7}$ Service de Médecine Intensive Réanimation, Centre Hospitalier Universitaire de Bicêtre, Le Kremlin-Bicetre, France

${ }^{8}$ Service de Réanimation et USC Médico-Chirurgicale, APHP.Sorbonne University, Tenon Hospital, Paris, France

${ }^{9}$ Service de Réanimation médicale, Centre Hospitalier de Mulhouse, Mulhouse, France

${ }^{10}$ Service de Réanimation et Surveillance Continue, Groupement Hospitalier Nord Essonne, Longjumeau, Île-de-France, France

${ }^{11}$ Service de Médecine intensive Réanimation, Groupe Hospitalier Paris CentreHôpital Cochin, Paris, Île-de-France, France

${ }^{12}$ Service de Médecine intensive et Réanimation, CHU de Brest, Brest, France

${ }^{13}$ Service de Médecine intensive Réanimation, CHU Dijon, Dijon, France

${ }^{14}$ Service de Médecine Intensive Réanimation, APHP. Hôpitaux Universitaires Henri Mondor, Creteil, France

Correction notice Armand Mekontso Dessap has been corrected.

Twitter Jean-Pierre Quenot @Quenot

Contributors MD, CE and AD in collaboration with all authors and the REVA network designed the study and wrote the manuscript together. CE provided substantial contributions to the conception and design of the study, and wrote the statistical analysis plan and estimated the sample size. MD, CE, AD, J-LB, FB, JC, $A G, L G, V L, G L, J L, J-P M, G P$ and J-PQ contributed for drafting the work, revising it critically for important intellectual content and approved the final version of the manuscript. MD, CE, AD, J-LB, FB, JC, AG, LG, VL, GL, JL, J-PM, GP and J-PQ give their agreement to be accountable for all aspects of the work, and ensure the accuracy and integrity of any part of the work.

Funding This study was funded by a grant from the French Ministry of Health obtained in 2018 (Programme Hospitalier de Recherche Clinique). The sponsor is Assistance Publique-Hôpitaux de Paris, AP-HP (Délégation à la Recherche Clinique et à l'Innovation, DRCI).

Competing interests MD reports financial support (expertise fees and travel expenses coverage to attend scientific meetings) by Lungpacer Med. Inc.

Patient and public involvement Patients and/or the public were not involved in the design, or conduct, or reporting, or dissemination plans of this research.

Patient consent for publication Obtained.

Provenance and peer review Not commissioned; externally peer reviewed.

Open access This is an open access article distributed in accordance with the Creative Commons Attribution Non Commercial (CC BY-NC 4.0) license, which 
permits others to distribute, remix, adapt, build upon this work non-commercially, and license their derivative works on different terms, provided the original work is properly cited, appropriate credit is given, any changes made indicated, and the use is non-commercial. See: http://creativecommons.org/licenses/by-nc/4.0/.

\section{ORCID iDs}

Martin Dres http://orcid.org/0000-0001-9191-6089

Guylaine Labro http://orcid.org/0000-0002-2578-3422

Jean-Pierre Quenot http://orcid.org/0000-0003-2351-682X

\section{REFERENCES}

1 Thille AW, Richard J-CM, Brochard L. The decision to extubate in the intensive care unit. Am J Respir Crit Care Med 2013;187:1294-302.

2 Liu J, Shen F, Teboul J-L, et al. Cardiac dysfunction induced by weaning from mechanical ventilation: incidence, risk factors, and effects of fluid removal. Crit Care 2016;20:369.

3 Bedet A, Tomberli F, Prat G, et al. Myocardial ischemia during ventilator weaning: a prospective multicenter cohort study. Critical Care 2019;23:321.

4 Frutos-Vivar F, Ferguson ND, Esteban A, et al. Risk factors for extubation failure in patients following a successful spontaneous breathing trial. Chest 2006;130:1664-71.

5 Upadya A, Tilluckdharry L, Muralidharan V, et al. Fluid balance and weaning outcomes. Intensive Care Med 2005;31:1643-7.

6 Silversides JA, Fitzgerald E, Manickavasagam US, et al. Deresuscitation of patients with iatrogenic fluid overload is associated with reduced mortality in critical IIIness ${ }^{*}$. Crit Care Med 2018;46:1600-7

7 National Heart, Lung, and Blood Institute Acute Respiratory Distress Syndrome (ARDS) Clinical Trials Network, Wiedemann HP, Wheeler AP, et al. Comparison of two fluid-management strategies in acute lung injury. N Engl J Med 2006;354:909-75.

8 Grams ME, Estrella MM, Coresh J, et al. Fluid balance, diuretic use, and mortality in acute kidney injury. CJASN 2011;6:966-73.

9 Marik PE, Linde-Zwirble WT, Bittner EA, et al. Fluid administration in severe sepsis and septic shock, patterns and outcomes: an analysis of a large national database. Intensive Care Med 2017;43:625-32.

10 Hjortrup PB, Haase N, Bundgaard H, et al. Restricting volumes of resuscitation fluid in adults with septic shock after initial management: the classic randomised, parallel-group, multicentre feasibility trial. Intensive Care Med 2016;42:1695-705.

11 Mekontso Dessap A, Roche-Campo F, Kouatchet A, et al. Natriuretic peptide-driven fluid management during ventilator weaning: a randomized controlled trial. Am J Respir Crit Care Med 2012;186:1256-63.

12 Dres M, Teboul J-L, Monnet X. Weaning the cardiac patient from mechanical ventilation. Curr Opin Crit Care 2014;20:493-8.

13 Thille AW, Muller G, Gacouin A, et al. Effect of Postextubation high-flow nasal oxygen with noninvasive ventilation vs high-flow nasal oxygen alone on reintubation among patients at high risk of extubation failure. JAMA 2019;322:1465.

14 Teboul J-L. Weaning-induced cardiac dysfunction: where are we today? Intensive Care Med 2014;40:1069-79.

15 Dres M, Teboul J-L, Anguel N, et al. Extravascular lung water, $B$-type natriuretic peptide, and blood volume contraction enable diagnosis of weaning-induced pulmonary edema. Crit Care Med 2014;42:1882-9.

16 Mekontso-Dessap A, de Prost N, Girou E, et al. B-Type natriuretic peptide and weaning from mechanical ventilation. Intensive Care Med 2006;32:1529-36.

17 Caille V, Amiel J-B, Charron C, et al. Echocardiography: a help in the weaning process. Critical Care 2010;14:R120.

18 Lamia B, Maizel J, Ochagavia A, et al. Echocardiographic diagnosis of pulmonary artery occlusion pressure elevation during weaning from mechanical ventilation*. Crit Care Med 2009;37:1696-701.

19 Quintard H, l'Her E, Pottecher J, et al. Experts' guidelines of intubation and extubation of the ICU patient of french society of anaesthesia and intensive care medicine (SFAR) and Frenchspeaking intensive care society (SRLF). Ann Intensive Care 2019;9:13.

20 Boles J-M, Bion J, Connors A, et al. Weaning from mechanical ventilation. Eur Respir J 2007;29:1033-56.

21 Hernández G, Vaquero C, Colinas L, et al. Effect of postextubation high-flow nasal cannula vs noninvasive ventilation on reintubation and postextubation respiratory failure in high-risk patients. JAMA 2016;316:1565-74.

22 Leone M, Bouadma L, Bouhemad B, et al. Hospital-acquired pneumonia in ICU. Anaes Crit Care \& Pain Med 2018;37:83-98.

23 Béduneau G, Pham T, Schortgen F, et al. Epidemiology of weaning outcome according to a new definition. the wind study. Am J Respir Crit Care Med 2017;195:772-83.

24 Dres M, Teboul J-L, Anguel N, et al. Passive leg raising performed before a spontaneous breathing trial predicts weaning-induced cardiac dysfunction. Intensive Care Med 2015;41:487-94.

25 Shen Y, Zhang W, Shen Y. Early diuretic use and mortality in critically ill patients with vasopressor support: a propensity score-matching analysis. Critical Care 2019;23:9.

26 McCoy IE, Montez-Rath ME, Chertow GM, et al. Estimated effects of early diuretic use in critical illness. Crit Care Explor 2019;1:e0021.

27 Martin GS, Mangialardi RJ, Wheeler AP, et al. Albumin and furosemide therapy in hypoproteinemic patients with acute lung injury*. Crit Care Med 2002;30:2175-82.

28 Vincent J-L, Sakr Y, Sprung CL, et al. Sepsis in European intensive care units: results of the soap study*. Crit Care Med 2006;34:344-53

29 Monnet X, Cipriani F, Camous L, et al. The passive leg raising test to guide fluid removal in critically ill patients. Ann Intensive Care 2016;6:46.

30 Hernández G, Vaquero C, González P, et al. Effect of postextubation high-flow nasal cannula vs conventional oxygen therapy on reintubation in low-risk patients. JAMA 2016;315:1354-61. 